\title{
Dynamics of Ordering in Alloys with Modulated Phases
}

\author{
Bulbul Chakraborty \\ The Martin Fisher School of Physics \\ Brandeis University \\ Waltham, MA 02254, USA
}

(August 15, 2018)

\begin{abstract}
This paper presents a theoretical model for studying the dynamics of ordering in alloys which exhibit modulated phases. The model is different from the standard time-dependent Ginzburg-Landau description of the evolution of a non-conserved order parameter and resembles the Swift-Hohenberg model. The early-stage growth kinetics is analyzed and compared to the Cahn-Hilliard theory of continuous ordering. The effects of non-linearities on the growth kinetics are discussed qualitatively and it is shown that the presence of an underlying elastic lattice introduces qualitatively new effects. A lattice Hamiltonian capable of describing these effects and suitable for carrying out simulations of the growth kinetics is also constructed.

64.60.Cn,5.50.+q,82.20.Mj,64.60.My
\end{abstract}

Typeset using REVTEX 


\section{INTRODUCTION}

One of the most fascinating problems in statistical mechanics is that of growth kinetics [1]; the evolution of order following a quench from an initial high temperature state to one below the transition temperature. Theoretical studies of kinetics have mainly concentrated on understanding the behavior of models such as the time-dependent Ginzburg-Landau model [2] or the kinetic Ising model [3]. Binary alloys have been viewed as convenient model systems for experimental investigation of these theoretical predictions [4, [5]. Many alloys, however, exhibit complex ordered phases including long-period, modulated structures [6] which cannot be described by simple antiferromagnetic Ising models [7]. The kinetics of ordering in these alloys should be significantly different from the usual models of ordering. These alloys can in turn provide models for both theoretical and experimental studies of a different class of kinetic phenomena.

This paper presents model partial-differential equations for describing growth kinetics in alloys with modulated phases and analyzes the early stage kinetics. The kinetics of growth in the strongly non-linear regime have to be approached through solutions of the partial differential equations or through Monte Carlo simulation of a discrete lattice Hamiltonian. Such a discrete Hamiltonian, derived from the same atomistic description that leads to the partial differential equations, is also presented, and the qualitative aspects of this model are discussed.

In recent years, there has been a concerted effort to understand phase stability in metallic alloys based on a realistic description of the "electron glue" responsible for metallic cohesion. One such approach, which has recently been applied to equilibrium phase transitions in the $\mathrm{Cu}-\mathrm{Au}$ alloys with success [8.9], is based on the effective medium theory (EMT) of cohesion in metals 10. EMT provides a semi-empirical scheme for writing down the energy of a system of electrons and ions with an arbitrary configuration. This is its major advantage over first-principles approaches which have to rely on specific symmetries of the system such as a) completely random [11] or b) ordered periodic structures [12. By its very nature, 
EMT is more approximate than the first-principles approaches; however, it does provide a model Hamiltonian which takes into account the metallic character of these alloys, describes the coupling of positional and configuration degrees of freedom [8,9] and can deal with size effects arising from the fact that the Ising spins are actually atoms which have different "sizes".

The EMT expression for the total energy for a given configuration of ions provides the classical Hamiltonian describing the alloys [8,9]. This Hamiltonian is a function of both the positions of the atoms and their chemical identity. The latter can be represented by an Ising variable [13]. So far, our studies of alloys have been based on an EMT Hamiltonian which includes the Ising variables and uniform lattice distortions, ie volume and shape distortions have been included but not volume conserving phonons [8,9]. From studies of compressible Ising systems, it is known that the coupling to uniform lattice distortions can change the nature of the Ising phase transition [14, 15]. The most striking demonstration of this is an Ising antiferromagnet on a deformable triangular lattice where the system was found to order, through a first-order phase transition, into a striped phase accompanied by a lattice distortion which has the antiferromagnetic bonds shortened and the ferromagnetic bonds lengthened [16]. On a rigid lattice there is no ordering. Our studies of the ordering in $C u A u$, based on EMT with uniform lattice distortions, showed that including the lattice distortions leads to a first-order transition from the disordered, undistorted lattice to a "layered" phase where the face-centered-cubic (fcc) lattice has alternating $\mathrm{Cu}$ (spin up) and $\mathrm{Au}$ (spin down) planes stacked along the (100) direction and there is a tetragonal distortion (the $L 1_{0}$ structure observed experimentally [17]) which shortens the antiferromagnetic bonds and stretches the ferromagnetic bonds [8,9]. This observation implies that the $C u A u$ transition is in the same class as the triangular lattice Ising antiferromagnet coupled to uniform lattice distortions. Part of the physics entering the EMT Hamiltonian for $\mathrm{Cu}-\mathrm{Au}$ can therefore be understood simply in terms of Ising antiferromagnets on frustrated but deformable lattices. The advantage of EMT is that the model is derived from a microscopic description of a given alloy and all the alloy-specific parameters are known. However, the mapping of the physics 
onto a simple model helps in understanding the physics of ordering in these alloys.

The other ingredient in EMT which is different from the usual Ising model description of alloys is the size of an atom. This size is defined in EMT as that radius of a sphere within which each atom is neutral. The size is therefore a configuration dependent quantity which depends on screening in a metallic environment. It has been argued [8] that the modulated CuAuII phase [17] is a consequence of such size effects and it will be shown here that these size effects lead to Ising models with competing interactions similar to ANNNI models [7]. The CuAuII phase has a one-dimensional modulation of the order parameter along a cubic direction and the period of the modulation, which varies as a function of composition, is ten lattice constants at the 50-50 composition. There is an alternate mechanism which could lead to modulated phases in metallic alloys, and this is driven by Fermi-surface instabilities [11. EMT, in its present form, cannot describe subtle Fermi surface effects and may fail to describe modulated phases in alloys where this is the dominant effect. The model of kinetics that will be presented in this paper can encompass both classes of modulated-phase alloys. The model will be derived for size-effect alloys such as $C u A u$ and the differences and similarities with Fermi-surface alloys will be discussed.

\section{MODEL OF KINETICS}

Pattern formation and kinetics of phase transitions are traditionally described by the time-dependent Ginzburg-Landau model [2]. For a non-conserved scalar order parameter $(\psi)$, this model Langevin equation can be written as,

$$
\frac{\partial \psi}{\partial t}=-\frac{\delta \mathcal{F}}{\delta \psi}+\eta(\boldsymbol{r}, t)
$$

where $\mathcal{F}[\psi]$ is the Ginzburg-Landau free energy functional, and $\eta(r, t)$ is a Gaussian noise term reflecting fluctuations of the heat bath [1,2]:

$$
\begin{aligned}
<\eta(\boldsymbol{r}, t) & >=0 \\
<\eta(\boldsymbol{r}, t) \eta\left(\boldsymbol{r}^{\prime}, t^{\prime}\right)> & =k_{B} T \delta\left(\boldsymbol{r}-\boldsymbol{r}^{\prime}\right) \delta\left(t-t^{\prime}\right) .
\end{aligned}
$$


It should be noted that $\mathcal{F}[\psi]$ also acts as a Lyapunov functional; $\frac{\partial \mathcal{F}[\psi(\boldsymbol{r}, t)]}{\partial t} \leq 0$.

Most descriptions of kinetics in alloys have been based on the traditional $\psi^{4}$ form of $\mathcal{F}[\psi]$. In contrast, our discussion of kinetics will be based upon a $\mathcal{F}[\psi]$ derived from the microscopic EMT Hamiltonian.

We use as our prototype alloy the $C u A u(50-50)$ alloy which exhibits modulated phases and, in equilibrium, has a first-order phase transition accompanied by a lattice distortion. The latter shows the relevance of the coupling to phonons and uniform lattice distortions and raises questions about the description of metastable and unstable states and the concept of a spinoidal. The presence of two first order transitions, from the disordered to the modulated $C u A u I I$ phase, followed by the transition from $C u A u I I$ to the zero temperature unmodulated $C u A u I$ structure [8,9], leads to unusual quench-temperature dependence of the kinetics and a Swift-Hohenberg-like [18] description of the early-stage kinetics.

The free-energy functional $\mathcal{F}[\psi]$ for the $C u A u$ alloy has been constructed by numerically calculating the mean-field free energy from the EMT Hamiltonian [8] and we will only briefly discuss the procedure and the results in this paper. The Hamiltonian includes uniform lattice distortions and the Ising degrees of freedom, $H\left(\left\{e_{\alpha}\right\},\left\{s_{i}\right\}\right)$. Where $s_{i}$ are the Ising variable on lattice sites $i$, and the $\left\{e_{\alpha}\right\}$ are the set of uniform lattice distortions. The partition function for this model can be written as

$$
Z=\int \Pi_{\alpha} d e_{\alpha} \int \mathcal{D} \psi \exp \left(-F\left[\psi, e_{\alpha}\right]\right)
$$

$i e$, one integrates over the lattice distortions and the order parameter field $\psi$. The GinzburgLandau functional $\mathcal{F}[\psi]$ is obtained by making a saddle-point approximation to the $e_{\alpha}$ integrals, ie minimizing $F$ with respect to these variables. In our numerical procedure, the mean-field free energy corresponding to a given set of $\psi$ and a given set of $e_{\alpha}$ was calculated from the EMT hamiltonian and this function was then minimized with respect to the $e_{\alpha}$. This is equivalent to making the magnetothermomechanics [19] approximation. The minimization of $\mathcal{F}[\psi]$ with respect to $\psi$ leads to the mean-field description of the $C u A u$ ordering transitions [8]. In this paper, this Ginzburg-Landau functional is used to describe 
the kinetics of phase transitions in these alloys.

The form of $\mathcal{F}[\psi]$ for $C u A u$, is most conveniently written in momentum space [20]:

$$
\begin{aligned}
\mathcal{F}[\psi] & =\sum_{\boldsymbol{q}}\left[a\left(T-T_{0}\right)+\omega(\boldsymbol{q})\right] \psi_{\boldsymbol{q}} \psi_{-} \boldsymbol{q} \\
& +\frac{u^{\prime}}{4} \sum_{\boldsymbol{q}} \psi_{\boldsymbol{q}_{1}} \ldots \psi_{\boldsymbol{q}_{4}} \delta\left(\boldsymbol{q}_{1}+\boldsymbol{q}_{2}+\boldsymbol{q}_{3}+\boldsymbol{q}_{4}\right) \\
& -\frac{u}{4}\left(\sum_{\boldsymbol{q}} \psi_{\boldsymbol{q}} \psi_{-\boldsymbol{q}}\right)^{2} \\
& +\frac{v}{6}\left(\sum_{\boldsymbol{q}} \psi_{\boldsymbol{q}_{1}} \psi_{\boldsymbol{q}_{2}} \ldots \psi_{\boldsymbol{q}_{6}} \delta\left(\boldsymbol{q}_{1}+\ldots \boldsymbol{q}_{6}\right)\right)
\end{aligned}
$$

where

$$
\omega(\boldsymbol{q})=e q_{z}^{2}+\left(q_{\perp}^{2}-q_{0}^{2}\right)^{2}-q_{0}^{4}
$$

Here $q_{\perp}$ is the magnitude of a wavevector in the plane perpendicular to the $C u A u$ ordering which is taken to be in the $z$-direction.

Before discussing the model of kinetics that follows from this Ginzburg-Landau functional, we briefly discuss the applicability of this model to equilibrium phase transitions in $C u A u$. Because of the form of $\omega(\boldsymbol{q})$, the Ginzburg-Landau functional, $\mathcal{F}[\psi]$, is minimized by a one-dimensional periodic function, which describes a configuration comprised of a periodic array of antiphase boundaries separating regions with $\psi= \pm 1$. This is the source of the CuAuII phase. The appearance of stationary one-dimensional, periodic patterns is also a feature of the Lyapunov functional associated with the Swift-Hohenberg equation [18. The isotropic form of $\omega(\boldsymbol{q})$ implies that the one-dimensional pattern is rotationally invariant in both models. However, the CuAuII structure observed in Monte Carlo simulations [8,9], based on EMT, and in experiments, has the modulation locked along one of the cubic axes, and there are lattice distortions accompanying this transition. The unusual fourth order term ( $3^{\text {rd }}$ term in Eq. (四)) arises from the coupling to uniform lattice distortions. This term is responsible for the $C u A u I$ type ordering [8,9] which, as discussed earlier, is in the same class as the Ising antiferromagnet on an elastic triangular lattice [16]. This type of ordering involves two broken symmetries, one corresponding to the twofold Ising degeneracy and one 
corresponding to the three possible strain directions on the lattice. The CuAuII ordering involves a lattice distortion along the direction of the one-dimensional modulation, and an additional symmetry breaking in the choice of this direction. It is expected that the coupling to the lattice is the source of the commensurate $C u A u I I$ ordering. In the Landau theory, presented earlier [8], the modulation was assumed to be along one of the cubic directions. The effects leading to the choice of this direction, within mean-field theory, are currently being investigated. It is clear from our Monte Carlo simulations that the EMT hamiltonian can describe the lattice distortions accompanying the modulation [8,9].

The function $\omega(\boldsymbol{q})$ can be measured in diffuse scattering experiments 21,22, The EMT model would predict an isotropic distribution in the plane perpendicular to the ordering direction ( $c f$ form of $\omega(\boldsymbol{q})$ in Eq. (田) ) and this isotropic distribution would have a peak displaced from the CuAuI superlattice Bragg peak by a distance $q_{0}$. Experimental measurements on $C u A u$ show an essentially isotropic distribution with a peak at $q_{0} \simeq 2 \pi / 10 a$ where $a$ is the lattice constant [21]. The EMT estimate for $q_{0}$ is $\simeq 2 \pi / 4 a$ [8]. This was, however, a very crude numerical estimate which was only meant to show that $q_{0}$ is nonzero. The essentially isotropic distribution of $C u A u$ is in sharp contrast with diffuse scattering intensities in $C u_{3} P d$ [22] where the distribution has well defined peaks along the $q_{x}$ and $q_{y}$ directions. These peaks have been explained on the basis of a Fermi-surface mechanism [23]. The essentially isotropic distribution in $C u A u$ suggests that a different mechanism is responsible for the modulated phase. The small anisotropy seen in experimental diffuse scattering measurements in $C u A u$ would be difficult to obtain from our numerical calculations based on EMT. It could also be that there is a Fermi surface contribution which is missing from EMT that makes the distribution anisotropic. Because of the small anisotropy, it is reasonable to investigate the isotropic model and discuss the consequences of the anisotropy. The isotropic model is interesting in its own right since it implies an the breaking of a continuous symmetry at the CuAuII transition, where the system chooses a direction of modulation.

The above discussion shows that $\mathcal{F}[\psi]$ is a good model for describing kinetics of these alloys. The kinetic equation derived from the model $\mathcal{F}[\psi]$, is 


$$
\frac{\partial \psi(\boldsymbol{r}, t)}{\partial t}=-\left\{\left[a\left(T-T_{0}\right)-q_{0}^{4}\right]-\frac{\partial^{2}}{\partial z^{2}}+\left(q_{0}^{2}+\nabla_{\perp}^{2}\right)^{2}\right\} \psi(\boldsymbol{r}, t)-\frac{\delta f}{\delta \psi}+\eta(\boldsymbol{r}, t)
$$

where

$$
f[\psi]=\frac{u^{\prime}}{4} \int \psi^{4}(\boldsymbol{r}) d \boldsymbol{r}-\frac{u}{4}\left(\int \psi^{2}(\boldsymbol{r}) d \boldsymbol{r}\right)^{2}+\frac{v}{6} \int \psi^{6}(\boldsymbol{r}) d \boldsymbol{r}
$$

\section{EARLY STAGE KINETICS}

The early stage kinetics of $C u A u$, following a quench, is defined by a linear stability analysis [24] of the disordered, $\psi=0$ state. This implies neglecting the $\delta f / \delta \psi$ term in Eq. (5). The linear theory is very different from the usual model of a non-conserved order parameter [24,25]. The difference is evident from an analysis of the solution to the linearized equations without the noise term. The easiest route to this solution is through transformation to momentum space where the different modes decouple and the solutions for $\psi(\boldsymbol{q})$ are,

$$
\psi(\boldsymbol{q}, t)=\exp (D(\boldsymbol{q}) t)
$$

where $D(\boldsymbol{q})=-a\left(T-T_{0}\right)-\omega(\boldsymbol{q})$. This solution shows that modes with $D(\boldsymbol{q})$ greater than zero grow exponentially with time. Eq. (6) is the generalization of the Cahn-Hilliard solutions describing continuous ordering [25] to alloys with modulated phases. If none of the eigenvalues, $D(\boldsymbol{q})$, are greater than zero then there is no continuous ordering and the disordered state is either stable or metastable. In contrast to the Cahn-Hilliard solutions, the fastest growing mode has a finite wavevector, $q_{0}$ and the growth rate depends only on the magnitude of the wavevector and not its direction. This is reminiscent of Cahn-Hilliard description of spinodal decomposition but there the wavevector of maximum growth depends on the temperature [1,25] whereas in our model, the linear dynamics chooses a unique length scale which is a characteristic of the system. Since the interesting pattern formations take place in the plane perpendicular to $q_{z}$, we will analyze the equations in the $q_{z}=0$ plane. This linearized $C u A u$ equation is then identical to the linearized version of the two-dimensional, stochastic Swift-Hohenberg equation [26]; 


$$
\frac{\partial \psi(\boldsymbol{r}, t)}{\partial t}=\left\{\epsilon-\left(1+\nabla_{\perp}^{2}\right)^{2}\right\} \psi(\boldsymbol{r}, t)
$$

if the control parameter $\epsilon$ is identified with $q_{0}{ }^{4}-a\left(T-T_{0}\right)$ and the natural wavevector $q_{0}$ is set to 1. The Swift-Hohenberg equation is used to model pattern-formation in Rayleigh-Benard convection and has been studied extensively both in one and two dimensions [26].

The linear dispersion relation $(D(q))$ is plotted in Fig. (1) for various temperatures. Three different temperature regimes can be identified. For $T \geq T_{0}+q_{0}{ }^{4} / a$, all modes decay and there is no continuous ordering, this is the regime where the disordered state is metastable and ordering takes place by nucleation. For $\left(T_{0}+q_{0}{ }^{4}\right)<T<T_{0}$, a band of wavevectors centered around $q_{0}$ grows and at $T=T_{0}$, the $q=0$ mode becomes unstable. The $q=0$ structure corresponds to the unmodulated $C u A u I$ phase. In the Cahn-Hilliard description of spinodal decomposition, the growth rate at $q=0$ is always zero, and in its description of continuous ordering, the $q=0$ mode always has the fastest growth rate. It should be remembered that the linear dispersion relation is isotropic in the plane perpendicular to $q_{z}$ and all modes with a given magnitude of $q$ grow at the same rate. This is expected to give rise to an interesting morphology of the early stage ordering process [27].

Experimental sudies of kinetics are based on the structure factor. The structure factor obtained from the linearized equation at a temperature within the second temperature regime is shown in Fig. (2a). The plot shows the evolution of the structure factor as a function of time. The peak of the structure factor remains stationary as as a small band of wavevectors centered around this peak grows as a function of time. The modes outside this band are seen to decay with the growth of order. At a lower temperature, the structure factor at $q=0$ would start to grow, but the growth rate predicted by the linear theory always has its maximum at $q=q_{0}$. The inclusion of the Gaussian noise term changes the form of the structure factor (generalization of the Cahn-Hilliard-Cook theory [25]) and can be written as

$$
S(\boldsymbol{q}, t)=S_{0}(q) \exp (2 D(\boldsymbol{q}) t)+\frac{k_{B} T}{2 D(\boldsymbol{q})}(\exp (2 D(\boldsymbol{q}) t)-1)
$$


where $S_{0}(q)$ is the structure factor in equilibrium at the temperature prior to the quench. The inclusion of the Cook term introduces minor modifications of the structure factor which are illustrated in Fig. (2b). These plots show that an experimental investigation of the early stage kinetics of $C u A u$ in this temperature regime should show a peak growing at a finite wavevector (measured with respect to the superlattice Bragg peak) and there should be a ring of wavevectors at which the structure factor is a maximum. The question of whether the early-stage description is ever valid in these systems can only be answered after a detailed study of the complete non-linear equations or from Monte Carlo simulations of the appropriate model Hamiltonian. It has been argued that the length of time over which the early stage kinetics remains valid is given approximately by $1 / D\left(q_{0}\right)$ and depends logarithmically on the range of interaction [28]. In our model, we have an effective infiniterange four-spin interaction arising from the coupling to the lattice distortions and this might imply that the range of validity of early-stage kinetics will be significantly enhanced in these systems. The evolution of the lattice distortion as a function of time is a novel feature of these systems and experimental investigations should shed light on the coupling between the evolution of order and the evolution of the lattice distortions.

\section{DISCUSSION OF NONLINEAR EFFECTS}

The effect of nonlinearities may change the kinetics in various ways. In the simplest case, the nonlinearities mainly come in to dampen the exponential growth. Some simple considerations of the nonlinearities in our model indicate that there can be more significant differences between the linear and nonlinear dynamics. The periodic solutions to which the $\psi=0$ state becomes linearly unstable have wavevectors lying in the range $\left[k_{1}, k_{2}\right]$, where

$$
k_{1,2}=\left(q_{0}^{2} \pm \sqrt{\left(q_{0}^{4}-a\left(T-T_{0}\right)\right)}\right)^{1 / 2}
$$

For temperatures close to but less than $T_{1}=T_{0}+q_{0}{ }^{4} / a$, the width of the band of wavevectors

grows as $\left|T-T_{1}\right|^{1 / 2}$. As the temperature approaches $T_{0}$, the $q=0$ mode becomes unstable 
and below $T_{0}$, the width grows as $\left(T-T_{0}\right)$ for $T$ close to $T_{0}$. This band of wavevectors, chosen by the linear dynamics, usually define the range in which the stationary periodic solution is found at a given temperature; ie, one could examine the periodic solutions lying in the range $\left[k_{2}, k_{1}\right]$, and the solution with the lowest free energy becomes the selected pattern [29]. However, because of the negative fourth order term in $\mathcal{F}[\psi]$, there is a first order transition from the disordered to the ordered periodic phase. This implies that, in a given temperature range, periodic solutions which are outside the band predicted by the linear dynamics could arise through a nucleated process and could become the stationary solutions. The condition that a order parameter of the form $\psi(\boldsymbol{r})=\psi \exp (i \boldsymbol{q} \cdot \boldsymbol{r})$ is a solution to

$$
\frac{\delta \mathcal{F}}{\delta \psi}=0
$$

is

$$
D(\boldsymbol{q}) \geq-\frac{\left(u-u^{\prime}\right)^{2}}{8 v}
$$

compared to the condition for linear stability, $D(\boldsymbol{q}) \geq 0$. For example, the $q=0$ state becomes the stable stationary solution at a temperature higher than its instability temperature $T_{0}$. The role of nonlinearities is different in this model than in the Swift-Hohenberg model. Since the negative fourth order term is a consequence of the coupling to the lattice [8.16], the investigation of non-linear dynamics has to take proper account of this coupling. The other crucial difference between this model and the Swift-Hohenberg model, is that the underlying lattice in $C u A u$ introduces a second length scale comparable to the length scale chosen by the linear dynamics $\left(2 \pi / q_{0}\right)$ and may be instrumental in locking in the direction of modulation. These effects are, in principle, all there in the nonlinear terms of our model equations if we are careful in keeping the Umklapp terms involving nonzero reciprocal lattice vectors in Eq. (田). However, it might be more profitable to investigate these effects directly in an effective lattice model.

Numerical solutions of the full non-linear Swift-Hohenberg equations have shown that the lamellar patterns exhibit phases which are akin to nematic and smectic ordering in liquid crystals 26]. In $C u A u$ alloys, the lamellar patterns have a length scale comparable to the 
underlying lattice and an analogy maybe drawn between these systems and nematic ordering in the presence of an underlying lattice [30]. The late stage growth laws in Swift-Hohenberg systems is expected to be different from usual models of non-conserved order parameters since the wavevector defining the modulation has a continuous symmetry [26]. It would be interesting to study the late stage growth laws in our model which has the extra feature of coupling to the lattice.

\section{LATTICE HAMILTONIAN}

We have argued that the study of non-linear dynamics is $C u A u$-like alloys may be best approached through a discrete lattice Hamiltonian. With this in mind, a lattice Hamiltonian has been constructed which has the salient features of the EMT Hamiltonian when applied to ordering on a deformable lattice [31]. The model is an Ising antiferromagnet on an elastic lattice with an unusual pair interaction term which arises from the size difference between the two chemical species making up the alloy. The source of this term in EMT is the density dependent cohesive energy [10,9]. When expanded in terms of the spin variables, a part of this energy gives rise to a term in the Hamiltonian which is an antiferromagnetic interaction between two atoms which share a common nearest neighbor 32. This interaction depends on the bond lengths and therefore can be different along different lattice directions in the presence of deformation. The strength of the antiferromagnetic interaction depends on the size-difference of the two atoms and is zero for atoms of the same size. This model can be written as,

$$
H=\sum_{\alpha} J\left(1-\epsilon e_{\alpha}\right) \sum_{<i j>_{\alpha}} s_{i} s_{j}+\sum_{\alpha \beta} M_{\alpha \beta} e_{\alpha} e_{\beta}+\sum_{\alpha} K \sum_{i}\left(\sum_{<i j>_{\alpha}} s_{j}\right)^{2} .
$$

The parameters can all be obtained from EMT, given a specific alloy, however, we will treat this as a general model with arbitrary parameters. This should then form a generic, minimal model for alloys where Fermi surface effects do not dominate. The first two terms in Eq. (10) describe an Ising model coupled to uniform strain through the coupling constant $\epsilon$ and an elastic Hamiltonian defined by the tensor $M_{\alpha \beta}$. This model for $J>0$ has 
been analyzed on the triangular lattice [16] and gives rise to a first order transition from a disordered to a striped phase. The last term in the Hamiltonian is the unusual term arising from size effects. Since $K \geq 0$, this term favors a spin configuration where, for each site, the sum of the nearest neighbor spins along a given direction add up to zero. In terms of atoms, this says that when atoms of different sizes are present, it is favorable to have a big atom and a small atom as nearest neighbors. Neither an antiferromagnetic, nor a striped phase meets the optimum demand of the $K$ term and a mean-field argument shows that, on a triangular lattice, modulated phases occur beyond a critical value of $K$ [32]. This is reminiscent of the ANNNI model. The one crucial feature which distinguishes the current model from the ANNNI model is that all order-disorder transitions are first-order because of the coupling to the lattice and makes it a more attractive model for describing alloys. Qualitatively, the source of the modulation can be inferred from looking at the striped phase of the triangular lattice antiferromagnet which has alternating chains of up and down spins and the sum over nearest neighbor spins for each direction adds up to two. If a modulation is introduced along the ferromagnetic direction, such that after $\mathrm{N}$ spins, the spin up and spin down chains are interchanged, then the spins at the domain boundaries have nearest neighbor configurations which do satisfy the $K$ term requirement. The reason for the choice of the ferromagnetic direction has its origin in the preferred lattice distortion. Therefore

in spite of the $K$ term being isotropic, a unidirectional modulation occurs. This scenario is very similar to the $C u A u I I$ ordering and investigations on the fcc lattice are currently underway. At this stage, we would like to present this model as an interesting variation on competing interactions models and suggest that the study of kinetics of this model would lead to novel features which can be tested through experimental studies of alloys.

\section{CONCLUSION}

As mentioned at the beginning of the paper, there is a class of modulated alloys where the modulation is due to Fermi-surface effects [23]. In these alloys, the $q$-dependence of the 
second order term in $\mathcal{F}[\psi]$ is not isotropic; ie, $\omega(\boldsymbol{q})$ has well defined minima along directions chosen by the Fermi-surface nesting vectors. The linear dynamics in these alloys chooses a length scale and a set of directions and the symmetry breaking that takes place is not continuous but discrete, Ising like. Also, the role of the lattice distortions are expected to be minimal and therefore the nonlinearities may play a simpler role and the late stage kinetics may follow the same scaling laws as in the usual models.

A complete description of ordering kinetics in alloys has to take into account the effect of phonons and local lattice distortions. Allowing for fluctuations in bond lengths also leads to an additional size-effect derived term in the lattice Hamiltonian. Under certain circumstances, this can give rise to a long-range pair interaction [33. The EMT prediction for this strain-mediated pair interaction term and its effects on kinetics is currently being investigated.

In conclusion, we have presented a model for describing kinetics of ordering in alloys which have modulated phases. The linear theory shows that the kinetics is similar to that of Swift-Hohenberg models, but the nonlinear terms are distinctly different from the SwiftHohenberg equations. A lattice Hamiltonian which captures the essential features of the microscopic model of these alloys has been constructed and simulations based on this model should be helpful in understanding both the statics and dynamics of ordering in these alloys.

\section{ACKNOWLEDGEMENTS}

The author wishes to thank W. Klein, Karl Ludwig, Duane Johnson, Frank Pinski and Mohan Phani for many helpful conversations. This work was supported in part by the NSF grant DMR-9208084. 


\section{REFERENCES}

[1] J. D. Gunton, M. San Miguel, and Paramdeep S. Sahni, in Phase Transitions and Critical Phenomena, Vol. 8, eds., C. Domb and J. L. Lebowitz

[2] Gene F. Mazenko, in Far From Equilibrium Phase Transitions, ed. Luis Garrido. (Springer-Verlag, Berlin, 1988).

[3] M. K. Phani, J. L. Lebowitz, M. H. Kalos and O. Penrose, Phys. Rev. Lett. 45, 366 (1980).

[4] S. E. Nagler et al, Phys. Rev. Lett. 617181988

[5] K. F. Ludwig et al, Phys. Rev. Lett. 6118591988

[6] Modulated Structures, eds., J. M. Cowley et al, (AIP Conference Proc. No. 53), (AIP, New York, 1979)

[7] W. Selke in Alloy Phase Stability, eds., G. M. Stocks and A. Gonis, NATO ASI series (Kluwer Academic, Dordrecht, 1987)

[8] Bulbul Chakraborty and Zhigang Xi, Phys. Rev. Lett. 6820391992

[9] Zhigang Xi et al, J. Phys: Condens. Matter 4, 7191 (1992)

[10] K. W. Jacobsen, J. K. Norskov, and M. J. Puska, Phys. Rev. B 3574231987, K. W. Jacobsen, Comments Cond. Mat. Phys. 14, 129 (1988).

[11] B. L. Gyorffy, D. D. Johnson, F. J. Pinski, D. M. Nicholson, and G. M. Stocks, in Alloy Phase Stability, eds., G. M. Stocks and A. Gonis, NATO ASI series (Kluwer Academic, Dordrecht, 1987)

[12] A. Zunger, S. H . Wei, A. A. Mbaye, and L. G. Ferreira, Acta Met 36, 2239 (1989); S. H. Wei, A. A. Mbaye, L. G. Ferreira and Alex Zunger, Phys. Rev. B 3641631987 and A. Zunger in Statics and Dynamics of Alloy Phase Transitions eds., P. E. A. Turchi and A. Gonis, NATO ASI series (Kluwer, Dordrecht, 1993). 
[13] D. de Fontaine, in Solid State Phys. ed. H. Ehrenreich, F. Seitz, and D. Turnbull, (Academic, N. Y., 1979 ) 34, pg. 73; D. de Fontaine in Electronic Band Structure and its applications, ed. M. Yossouf, (Springer Verlag, Berlin, 1986) and references therein.

[14] D. J. Bergman and B. I. Halperin, Phys. Rev. B 1321451976.

[15] B. K. Chakrabarti et al, J. Phys. C 15, L777 (1982).

[16] Zhong-Ying Chen and Mehran Kardar, J. Phys. C 19, 6825 (1986) and references therein.

[17] W. B. Pearson, Handbook of Lattice Spacings and Structures of Metals, (Pergamon, N. Y., 1958); M. Hansen, Constitution of Binary Alloys, (McGraw Hill, N. Y. , 1958).

[18] J. Swift and P. C. Hohenberg Phys. Rev. A 15, 319 (1977)

[19] D. C. Mattis and T. D. Shulz Phys. Rev 129, 175 (1963).

[20] In writing this form it has been assumed that the wavevectors are much smaller that a reciprocal lattice vector, if this condition is not satisfied than the constraints imposed by the delta functions have to be replace by constraints where the $q$-vectors add up to a reciprocal lattice vector. These are the so-called Umklapp terms.

[21] S. Hashimoto, Acta Cryst A39, 524 (1983).

[22] K. Ohshima et al, Acta Cryst A32, 883 (1976).

[23] B. L. Gyorffy and G. M. Stocks, Phys. Rev. Lett. 50, 374 (1983)

[24] M. C. Cross in Far From Equilibrium Phase Transitions, ed. Luis Garrido. (SpringerVerlag, Berlin, 1988).

[25] W. Klein and G. G. Batrouni, Phys. Rev. Lett. 67, 1278 (1991).

[26] K. R. Elder, et al, Phys. Rev. A 46, 7618 (1992) and references therein.

[27] W. Klein, Phys. Rev. Lett. 65, 1462 (1990). 
[28] K. Binder, Phys. Rev. A 29, 341 (1984).

[29] Fong Liu and Nigel Goldenfeld, Phys. Rev. A 39, 4805 (1989).

[30] J. V. Selinger and David R. Nelson, Phys. Rev. A 39, 3135 (1989).

[31] The EMT Hamiltonian can describe alloy phases which are not on a lattice, here we look at a restricted Hamiltonian which describes alloy on a deformable lattice.

[32] Bulbul Chakraborty, in Proceedings of NATO ARW workshop on Metallic Alloys: Experimental and Theoretical Perspectives, eds. J. S. Faulkner and R. G. Jordan, (Boca Raton, Florida, July 16-23, 1993); M. Sobkowicz and B. Chakraborty, to be published

[33] H. Nishimori and A. Onuki, Phys. Rev. B 42, 980 (1990); S. Semenovskaya and A. Khachaturyan, Phys. Rev. Lett. 67, 2223 (1991), and references therein. 


\section{FIGURES}

FIG. 1. The linear dispersion relation $D(\mathbf{q})$ vs. $\left|q_{\perp}\right|$. The dispersion is isotropic in the $\left|\mathbf{q}_{\perp}\right|$ plane. Four temperatures are shown with the bottom most curve at the temperature $T_{1}=T_{0}+q_{0}{ }^{4} / a$ and the topmost curve at the temperature $T_{0}$.

FIG. 2. (a) The evolution in time of the structure factor obtained from the linear theory without the noise term. The plots show $S(\mathbf{q})$ at a temperature $T$ such that $T_{1} \leq T \leq T_{0}$. The crosses are at the earliest time and the squares at the latest time. (b) The evolution in time of the structure factor predicted by the linear theory with the noise term included. The parameters used are those appropriate for $\mathrm{CuAu}$. 